TITLE:

\title{
Reduced representational momentum for subtle dynamic facial expressions in individuals with autism spectrum disorders
}

\section{AUTHOR(S):}

Uono, Shota; Sato, Wataru; Toichi, Motomi

\section{CITATION:}

Uono, Shota ... [et al]. Reduced representational momentum for subtle dynamic facial expressions in individuals with autism spectrum disorders. Research in Autism Spectrum Disorders 2014, 8(9): 1090-1099

\section{ISSUE DATE:}

2014-09

URL:

http://hdl.handle.net/2433/189087

\section{RIGHT:}

(C) 2014 Elsevier Ltd.; This is not the published version. Please cite only the published version.; この論文は出版社版でありません。引用の際に は出版社版をご確認ご利用ください。 
Research in Autism Spectrum Disorders doi:10.1016/j.rasd.2014.05.018

1 Running Head: Representational momentum in ASD

3 Reduced representational momentum for subtle dynamic facial express ions in individuals with autism spectrum disorders

Shota Uono ${ }^{1}$, Wataru Sato $^{2}$, Motomi Toichi ${ }^{1,3}$

6

71 Graduate School of Medicine, Faculty of Human Health Science, Kyoto University,

853 Shogoin Kawahara-cho, Sakyo-ku, Kyoto 606-8507, Japan.

92 The Hakubi Project, Primate Research Institute, Kyoto University, Inuyama, Aichi

10 484-8506, Japan.

113 The Organization of Promoting Developmental Disorder Research, 40 Shogoin

12 Sannou-cho, Sakyo-ku, Kyoto 606-8392, Japan.

14 Correspondence concerning this article should be addressed to Shota Uono, Graduate

16 Kawahara-cho, Sakyo-ku, Kyoto 606-8507, Japan.

17 Tel: +81- 75-751-3965; Fax: +81-75-751-3965;

18 E-mail: uonoshota1982@gmail.com 
Research in Autism Spectrum Disorders doi:10.1016/j.rasd.2014.05.018

\section{Abstract}

21 The cognitive mechanisms underlying social communication via emotional facial

22 expressions are crucial for understanding the social impairments experienced by people

23 with autism spectrum disorders (ASD). A recent study (Yoshikawa \& Sato, 2008) found

24 that typically developing individuals perceived the last image from a dynamic facial expression to be more emotionally exaggerated than a static facial expression; this

26 perceptual difference is termed representational momentum (RM) for dynamic facial expressions. RM for dynamic facial expressions might be useful for detecting emotion in another's face and for predicting behavior changes. We examined RM for dynamic facial expressions using facial expression stimuli at three levels of emotional intensity

30 (subtle, medium, and extreme) in people with ASD. We predicted that individuals with

31 ASD would show reduced RM for dynamic facial expressions. Eleven individuals with

32 ASD (three with Asperger's disorder and eight with pervasive developmental disorder

33 not otherwise specified) and 11 IQ-, age- and gender-matched typically developing

34 controls participated in this study. Participants were asked to select an image that matched the final image from dynamic and static facial expressions. Our results revealed that subjectively perceived images were more exaggerated for the dynamic 
Research in Autism Spectrum Disorders doi:10.1016/j.rasd.2014.05.018

38 group, however, perceived a reduced degree of exaggeration for dynamic facial

39 expressions under the subtle intensity condition. As facial expressions are often

40 displayed subtly in daily communications, reduced RM for subtle dynamic facial

41 expressions may prevent individuals with ASD from appropriately interacting with

42 other people as a consequence of their difficulty detecting others' emotions.

43 Keywords: Autism spectrum disorders; Dynamic facial expression; Representational 
Research in Autism Spectrum Disorders doi:10.1016/j.rasd.2014.05.018

Reduced representational momentum for subtle dynamic facial expressions in

\section{individuals with autism spectrum disorders}

\section{Introduction}

Individuals with autism spectrum disorders (ASD) have difficulty with social

50 interaction, including communication via emotional facial expressions (American

51 Psychiatric Association [APA], 2000). Clinical observation studies have consistently

confirmed that individuals with ASD are impaired in many types of social interactions

involving facial expressions. For example, previous studies examining children's

behavior under structured conditions have demonstrated that individuals with ASD

exhibit reduced attention (Sigman, Kasari, Kwon, \& Yirmiya, 1992), emotional

behaviors (Corona, Dissanayake, Arbelle, Wellington, \& Sigman, 1998), and facial

reactions (Yirmiya, Kasari, Sigman, \& Mundy, 1989) in response to the facial

expressions of other individuals.

60 facial expressions in individuals with ASD, though findings still remain rather

61 inconsistent. Almost all of these studies have used static facial expressions as stimuli. In

62 some of these studies, individuals with ASD have shown more perturbation in the

63 ability to recognize facial expressions than typically developing individuals (Ashwin, 
Research in Autism Spectrum Disorders doi:10.1016/j.rasd.2014.05.018

64 Chapman, Colle, \& Baron-Cohen, 2006; Braverman, Fein, Lucci, \& Waterhouse, 1989;

65 Celani, Battacchi, \& Arcidiacono, 1999). However, other studies have failed to show

66 such impaired recognition (Adolphs, Sears, \& Piven, 2001; Castelli, 2005; Grossman,

67 Klin, Carter, \& Volkmar, 2000).

Everyday communication of emotions is largely based on dynamic facial cues.

69 Real-life facial expressions reflect dynamic, moment-to-moment changes in emotional

70 state (Ekman \& Friesen, 1975). A growing body of studies has consistently shown that

71 various psychological activities including subjective perception (Yoshikawa \& Sato,

72 2008), recognition (Ambadar, Schooler, \& Cohn, 2005; Bould \& Morris, 2008; Bould,

73 Morris, \& Wink, 2008), and emotional responses (Sato \& Yoshikawa, 2007a, 2007b)

74 are enhanced in response to dynamic expressions as compared with static facial

expressions. Neuroimaging studies have also demonstrated that some brain regions

76 show increased activity in response to dynamic, rather than static, facial expressions

77 (LaBar, Crupain, Voyvodic, \& McCarthy 2003; Sato, Kochiyama, Yoshikawa, Naito, \&

Matsumura, 2004). Taken together, these findings indicate that dynamic facial

expressions are more effective for emotional communication than are static facial

80 stimuli. These findings also suggest the possibility that individuals with ASD may have

81 more difficulty processing dynamic facial expressions than static facial expressions. 
Research in Autism Spectrum Disorders doi:10.1016/j.rasd.2014.05.018

To investigate the processing of dynamic facial expressions in individuals with

83 ASD, pioneering studies have examined their recognition of these stimuli (Gepner,

84 Deruelle, \& Grynfeltt, 2001; Tardif, Lainé, Rodriguez, \& Gepner, 2007). Gepner et al.

85 (2001) showed that a strobe presentation (i.e., presentation of a few frames of a clip

86 revealing changes in dynamic facial expressions, to produce the illusion of motion)

87 improved facial expression recognition as measured by a matching-to-sample task,

88 compared to static presentation, in typically developing controls but not in individuals

89 with ASD. However, individuals with ASD were able to recognize both dynamic and

90 static facial expressions. Using a similar matching-to-sample method, Tardif et al.

91 (2007) demonstrated that individuals with ASD were less able than typically developing

92 individuals to recognize dynamic and static facial expressions, but slowing down the

93 presentation of dynamic facial expressions improved their recognition. These studies

94 suggest differences in performance between individuals with ASD and typically

95 developing individuals in the recognition of dynamic facial expression. However,

96 dynamic presentation did not improve the recognition of facial expressions by typically

97 developing individuals in these studies. Recently, Kessels, Spee, and Hendriks (2010)

98 found that labeling of dynamic facial expressions, specifically those of fearful and

99 disgusted emotions, was defective in individuals with ASD. However, recognition 
Research in Autism Spectrum Disorders doi:10.1016/j.rasd.2014.05.018

100 involved several processing stages; these included perceptual processing, interpretation

101 of emotional meaning, and selection of an appropriate verbal label. Consequently, it is

102 difficult to reach definite conclusions about which of the stages involved in processing

103 dynamic facial expressions are impaired in individuals with ASD. To elucidate

104 impairments specific to ASD, it is necessary to use an experimental paradigm in which

105 dynamic presentation enhances the processing of facial expressions in typically

106 developing individuals and to examine each component of this dynamic facial

107 expression processing, such as the perception and interpretation of emotional meaning.

108 Functional magnetic resonance imaging (fMRI) studies have shown that

109 dynamic facial expressions elicit atypical neural activation in several brain regions of

110 ASD individuals (Pelphrey, Morris, McCarthy, \& Labar, 2009; Sato, Toichi, Uono, \&

111 Kochiyama, 2012). Pelphrey et al. (2007) presented dynamic and static facial

112 expressions depicting anger, fear, or neutral emotions, and found that observation of

113 dynamic facial expressions elicited less activation in the superior temporal

114 sulcus/middle temporal gyrus (STS/MTG), fusiform gyrus (FG), amygdala (AMY), and

115 medial prefrontal cortex (MPFC) in individuals with ASD compared to typically

116 developing individuals. Sato et al. (2012) extended these findings using happy and

117 fearful emotional stimuli. The results showed that, compared to the typically developing 
Research in Autism Spectrum Disorders doi:10.1016/j.rasd.2014.05.018

118 group, the ASD group exhibited less activation in the brain regions described above,

119 and also in the inferior frontal gyrus (IFG), in response to dynamic facial expressions.

120 These regions are involved in various aspects of processing of social stimuli, including

121 visual analysis of the dynamic aspects of faces (STS/MTG; Allison, Puce, \& McCarthy,

122 2000); visual analysis of the invariant aspects of faces; the subjective perception of

123 faces (FG; Haxby, Hoffman, \& Gobbini, 2000); emotional processing (AMY; Calder,

124 Lawrence, \& Young, 2001); attribution of mental states (MPFC; Frith \& Frith, 2003);

125 and motor mimicry (IFG; Iacoboni, 2005). It is tempting to speculate that deficits in

126 such psychological functions influence the processing of dynamic facial expressions in

127 individuals with ASD. However, as fMRI has inherent technical limitations in terms of

128 temporal resolution, and as the abovementioned brain regions are functionally and

129 structurally connected, it remains unclear which level or levels of processing are

130 impaired in the processing of dynamic facial expressions in individuals with ASD.

131 To investigate the more rapid components of dynamic facial expression

132 processing, Uono, Sato, and Toichi (2010) recently studied the subjective perception of

133 facial expressions in individuals with ASD. This study measured the representational

134 momentum (RM) of dynamic facial expressions. RM refers to a phenomenon in which

135 the perceived final position of a moving object shifts in the direction of the actually 
Research in Autism Spectrum Disorders doi:10.1016/j.rasd.2014.05.018

136 observed movement (Freyd \& Finke 1984; Hubbard, 1990). This effect has also been

137 reported in the perception of biological stimuli, including dynamic facial expressions

138 (Hudson, Liu, \& Jellema, 2009; Yoshikawa \& Sato, 2008). Uono et al. presented

139 dynamic or static facial expressions and asked participants to choose from a display of

140 variable emotional expressions the image that matched the final image from the

141 presented expression. In this task, dynamic presentation clearly enhanced processing of

142 facial expressions in typically developing individuals (Yoshikawa \& Sato, 2008).

143 Further, the task allowed the perceptual processing of dynamic facial expressions to be

144 investigated, because neither interpretation of emotional meaning nor selection of a

145 verbal label was required. Contrary to expectations, both those with and without ASD

146 perceived the final images from the dynamic facial expressions to be more emotionally

147 exaggerated than the static facial expressions. This finding suggests that individuals

148 with ASD have an intact ability to process dynamic information from facial cues, at

149 least on a perceptual level.

150 However, one limitation of that study was that only a single intensity level of

151 facial expression stimuli was used. The stimuli were at a facial expression intensity of

$15280 \%$ based on a standard set (Ekman \& Friesen, 1976) and showed clear to moderately

153 clear emotions. 
Research in Autism Spectrum Disorders doi:10.1016/j.rasd.2014.05.018

One important area for exploration involves studying subtle facial expressions.

155 In everyday communication, many facial expressions are displayed with subtle intensity

156 (Ekman, 2003; Motley \& Camden, 1988). Behavioral studies suggest that the detection

157 of subtle expressions provides an advantage in social interactions (e.g., Warren,

158 Schertler, \& Bull, 2009; Yoon, Joormann, \& Gotlib, 2009) because it allows us to notice

159 others' subtle emotional changes and to regulate our own behaviors appropriately.

160 Consistent with these notions, it has been suggested that dynamic information is more

161 important when processing subtle than when processing intense emotional expressions

162 (cf. Ambadar et al., 2005; Bould \& Morris, 2008; Bould et al., 2008). If RM for

163 dynamic facial expressions is an adaptive mechanism for detecting emotion, it is

164 assumed to play a particularly crucial role in processing subtle expressions. Thus, one

165 contributor to the social interaction difficulties of individuals with ASD may be

166 compromised processing of subtle dynamic facial expressions. We hypothesize that

167 individuals with ASD may perceive subtle dynamic facial expressions in a less

168 exaggerated form than typically developing individuals do.

169 Another possible avenue of exploration would be to use more extreme facial

170 expressions. In the previous study that tested RM in typically developing individuals

171 (Yoshikawa \& Sato, 2008), the researchers suggested that the effects of dynamic facial 
Research in Autism Spectrum Disorders doi:10.1016/j.rasd.2014.05.018

172 expressions tend to be weaker at $100 \%$ intensity compared with at $80 \%$ intensity.

173 Yoshikawa and Sato speculated that the RM for $100 \%$ expressions might be suppressed

174 because participants evaluate such expressions as extreme during realistic social

175 interactions. A recent behavioral study (Rutherford \& McIntosh, 2007) investigated the

176 perception of facial expressions varying in intensity in ASD. Rutherford and McIntosh

177 (2007) presented two emotional faces with different intensities and asked participants to

178 select the more realistic image. The results showed that individuals with ASD were

179 more likely to judge extremely exaggerated facial expressions as the most realistic.

180 Based on these findings, dynamic facial expressions of extremely high intensity might

181 trigger further changes in individuals with ASD, but not controls, whereas those of

182 medium intensity did so in both groups. Thus, we hypothesized that individuals with

183 ASD might perceive dynamic facial expressions as more exaggerated than normal

184 controls do under the highest intensity condition.

This study investigated RM for dynamic facial expressions at various intensities

186 among individuals with high-functioning ASD and IQ-, age-, and gender-matched

187 typically developing controls. We presented dynamic and static facial expressions at

188 subtle, medium, and extreme intensities and asked participants to change an emotional

189 face display to match the perceived final image from dynamic and static facial 
Research in Autism Spectrum Disorders doi:10.1016/j.rasd.2014.05.018

190 expression stimuli. Based on a previous study (Uono et al., 2010) and the

191 aforementioned evidence, we predicted that (1) both the ASD and control groups would

192 perceive the final images from the dynamic facial expressions to be more emotionally

193 exaggerated than the static facial expressions; (2) the ASD group would perceive subtle

194 dynamic facial expressions as less exaggerated than would the control group; and (3)

195 the ASD group, compared with the control group, would perceive dynamic facial

196 expression as more exaggerated under the extremely intense emotion condition.

197

198 2. Materials and methods

199 2.1 Participants

200 The participants were 11 individuals with ASD and 11 typically developing

201 controls. The two groups (ASD and control) were matched for chronological age (ASD

202 group: mean $\pm S D=22.1 \pm 4.8$; control: mean $\pm S D=22.8 \pm 2.5$; independent $t$-test,

$203 t(20)=0.46, p>0.10$ ), gender (ASD group: eight males, three females; control: seven

204 males, four females; Fisher's exact test, $p>0.10$ ) and IQ (mean $\pm S D$ verbal IQ, control:

$205117.1 \pm 10.9$, ASD: $113.1 \pm 10.2, t(20)=0.86, p>0.10 ;$ mean $\pm S D$ performance IQ,

206 control: $111.5 \pm 10.7$, ASD: $108.5 \pm 13.9, t(20)=0.57, p>0.10$; mean $\pm S D$ full-scale

207 IQ, control: $116.1 \pm 10.3$, ASD: $111.6 \pm 9.9, t(20)=1.03, p>0.10)$. Verbal and 
Research in Autism Spectrum Disorders doi:10.1016/j.rasd.2014.05.018

performance IQ was measured using the Japanese version of the WAIS-R (Shinagawa,

209 Kobayashi, Fujita, \& Maekawa, 1990), WAIS-III (Fujita, Maekawa, Dairoku, \&

210 Yamanaka, 2006), WISC-R (Kodama, Shinagawa, \& Motegi, 1982), and WISC-III

211 (Azuma et al., 1998). Handedness was assessed in individuals with and without ASD

212 using the Edinburgh Handedness Inventory (Oldfield, 1971). The scores did not differ

213 between groups (control: mean $\pm S D=80.0 \pm 53.7$; ASD: mean $\pm S D=80.0 \pm 41.0$;

214 independent $t$-test, $t(20)<0.01, p>0.10)$. Both groups included 10 right-handed and

215 one left-handed participants. All the participants had normal or corrected-to-normal

216 visual acuity.

The participants in the ASD group were diagnosed with either Asperger's

218 disorder (three males) or pervasive developmental disorder not otherwise specified

219 (PDD-NOS; five males and three females) at the time of the present study according to

220 the DSM-IV-TR criteria (APA, 2000). PDD-NOS includes heterogeneous subgroups of

221 PDD with varying degrees of qualitative social impairment. The participants with

222 PDD-NOS included in the present study did not satisfy criteria for Asperger's disorder

223 because 1) they had similar impairments in qualitative social interaction without

224 apparently restricted interests or stereotyped behaviors, or (2) their impairment in

225 qualitative social interaction was milder than that observed in Asperger's disorder. Thus, 
Research in Autism Spectrum Disorders doi:10.1016/j.rasd.2014.05.018

our participants with PDD-NOS had milder pathologies than did those with Asperger's

227 disorder. The final diagnoses were made by a child psychiatrist (MT) based on the

228 reports of clinical psychologists, interviews with each participant, information from each participant's parents or teachers, and childhood clinical records when available.

230 The participants in the ASD group were outpatients who had been referred to Kyoto

231 University Hospital or to the Division of Human Health Science of Kyoto University

232 Graduate School of Medicine due to social maladaptation. They were all free of neurological or psychiatric problems other than those derived from ASD, and none was

234 receiving any medication. The members of the typically developing control group were students at several universities who were recruited using paper- and web-based

236 advertisements. After acquiring the data from the ASD group, we collected IQ data

237 from typically developing participants. Eleven typically developing participants who

238 matched the ASD group in terms of age and IQ were selected for participation. The participants aged younger than 18 years received written informed consent from their

240 parents to participate in the study. The study was conducted in accord with institutional

241 ethical provisions and the Declaration of Helsinki. 
Research in Autism Spectrum Disorders doi:10.1016/j.rasd.2014.05.018

244 interviews with participants and their parents and direct observations of participants

245 during these interviews. The evaluations were performed by psychiatrists. The CARS

246 has been shown to be an effective tool for diagnosing autism in adolescents, adults, and

247 children (Mesibov, Schopler, Schaffer, \& Michal, 1989). The CARS scores of the ASD

248 group (mean $\pm S D=21.04 \pm 2.67$ ) were comparable to those of Japanese individuals

249 with Asperger's disorder in a previous study (mean $\pm S D=22.22 \pm 3.57 ; t(45)=1.01, p$

$250>0.10$ ) (Koyama, Tachimori, Osada, Takeda, \& Kurita, 2007). These data indicate that

251 the symptoms of individuals in the ASD group were severe enough to allow for the

252 diagnosis of ASD.

253

$254 \quad 2.2$ Design

255

The experiment was constructed as a three-factorial mixed randomized-repeated

256 design, with group (ASD or control) as the randomized factor and presentation

257

condition (dynamic or static) and intensity $(52 \%, 80 \%$, or $108 \%)$ as the repeated factors.

258

259

2.3 Stimuli

260

From a set of facial images (Ekman \& Friesen, 1976), we selected one neutral expression slide and two emotional expression (fearful and happy) slides for each of 
Research in Autism Spectrum Disorders doi:10.1016/j.rasd.2014.05.018

262 four actors (two men and two women). We used computer-morphing techniques

263 (Mukaida et al., 2000) to produce images that were intermediate between the neutral

264 expression and each of the two emotional expressions in 4\% steps. We produced

265 dynamic facial expression stimuli that changed from $4 \%$ emotional expression to a

266 maximum of $52 \%, 80 \%$, or $108 \%$ of the original emotional expression in $4 \%$ steps. To

267 create the images of $108 \%$ emotional expression, we changed the facial features of the

$268100 \%$ emotional expression in the direction opposite from that depicted in the neutral

269 face. We presented a total of 13, 20, and 27 image frames in succession for the $52 \%$,

$27080 \%$, and $108 \%$ conditions, respectively (e.g., under the $52 \%$ condition, the first image

271 was followed by 11 intermediate images changing from $8 \%$ to $48 \%$ in $4 \%$ steps, ending

272 with the final image). Under the dynamic condition, each frame was presented for $10 \mathrm{~ms}$.

273 Thus, the total presentation time was $130 \mathrm{~ms}, 200 \mathrm{~ms}$, and $270 \mathrm{~ms}$ for the $52 \%, 80 \%$,

274 and $108 \%$ conditions, respectively. Fig. 1 shows the first image, some intermediate

275 images, and the final image of a dynamic stimulus. Under the static condition, only the

276 last frame of each dynamic facial expression stimulus was presented. The total

277 presentation time was the same as that for the dynamic facial expression with the

278 corresponding intensity. 
Research in Autism Spectrum Disorders doi:10.1016/j.rasd.2014.05.018

2.4 Apparatus

Stimulus presentation and data acquisition were controlled using a program

written in Visual C++ 5.0 (Microsoft) on a Windows computer (HP xw4300

Workstation). Stimuli were presented on a 17-in CRT monitor (Iiyama; screen

resolution $1024 \times 768$ pixels; refresh rate $100 \mathrm{~Hz}$ ). The distance between the monitor and participants was fixed at approximately $57 \mathrm{~cm}$ using a headrest.

292 et al., 2010; Yoshikawa \& Sato, 2008). On the monitor, two windows were presented.

293 The left window was used for stimulus presentation, and the right window was used for

294 responses. The vertical and horizontal visual angles of the stimulus and response

295 windows were $11.1^{\circ}$ and $7.8^{\circ}$, respectively. In each trial, a cross hair was first presented

296 at the center of the stimulus window. The participants were instructed to fixate on this.

297 Then, a dynamic or static stimulus was presented in the stimulus window, and $250 \mathrm{~ms}$ 
Research in Autism Spectrum Disorders doi:10.1016/j.rasd.2014.05.018

298 later, an initial face image was presented in the response window. Participants were

299 instructed to match the image in the response window exactly with the last image shown

300 in the dynamic or static stimulus, by using the mouse to drag a slider to the left or right.

301 The face shown in the initial image in the response window had an emotional

302 expression with $-10 \%, 0 \%$, or $+10 \%$ intensity of the presented stimuli (e.g., under the

$30352 \%$ condition, $42 \%, 52 \%$, or $62 \%$ ). The upper or lower limit of the slide had one of

304 three predefined ranges, each of which covered an 80\% range of intensity (e.g., under

305 the $52 \%$ condition, $2-82 \%, 12-92 \%$, or $22-102 \%)$. The ranges of the scale varied

306 randomly across trials and were not visible to the participants. After a participant

307 selected an image, he or she clicked a button, and the image in the response window

308 disappeared. Then, the stimulus was presented again in the left window, and $250 \mathrm{~ms}$

309 later, the image chosen by the participant appeared in the response window. If the

310 participant thought the images matched, he or she clicked the button on the display and

311 went on to the next trial; if not, the participant could modify the image until he or she

312 thought it matched. No time limits were set for the first or second judgment. Before

313 starting the experiment, each participant was given several practice trials and allowed to

314 practice image manipulation using the mouse to move the slider. A total of 48 trials 
Research in Autism Spectrum Disorders doi:10.1016/j.rasd.2014.05.018

315 (eight trials per condition) were performed in blocks, and the order of trials was

316 counterbalanced across participants.

$318 \quad 2.6$ Data analysis Data were analyzed using SPSS10.0J (SPSS Japan). For each participant, the mean intensity of response images was calculated for each condition. Then, the ratio

321 between the intensity of responses and of presented images was calculated for each condition. The ratios were analyzed with a 2 (group) $\times 2$ (presentation) $\times 3$ (intensity) repeated-measures analysis of variance (ANOVA). To test our predictions, follow-up

324 simple interaction analyses and simple-simple main-effect analyses were conducted (cf.

325 Kirk, 1995).

327 dysfunction in individuals with ASD. As in our previous studies (Uono, Sato, \& Toichi,

328 2011; 2013), we used the following CARS items, which were classified as elements of

329 the social functioning construct: "imitation," " "nonverbal communication,"

330 "relationship to people," “verbal communication," and “"visual response." We

331 averaged the scores on these items to obtain the social dysfunction scale. To analyze the

332 relationships between degree of RM and CARS scores, the ratio between the mean 
Research in Autism Spectrum Disorders doi:10.1016/j.rasd.2014.05.018

333 intensity of the responses to images under dynamic and static conditions was calculated

334 for each intensity condition. Pearson's product-moment correlations between

335 combinations of these variables were calculated. The significance of correlation

336 coefficients was evaluated using $t$-tests (two-tailed). We excluded multivariate outliers

337 by calculating the Mahalanobis distance for each case $(p<0.10)$.

338

339 3. Results

340 The mean response under each condition (with $S E$ ) is shown in Table 1. The

341 ratios between the intensity of response images and presented images were calculated

342 (Fig. 2) and subjected to a group $\times$ presentation $\times$ intensity ANOVA. Most importantly,

343 the results revealed a significant three-way interaction $(F(2,40)=3.52, p=0.04)$.

344 Additionally, the results revealed a main effect of presentation $(F(1,20)=52.20, p<$

345 0.01), indicating that participants perceived more exaggerated images under dynamic

346 than under static conditions. A main effect of intensity was also found $(F(2,40)=26.75$,

$347 p<0.01)$. Other main effects and interactions were not significant $(F<2.37, p s>0.10)$.

****************************** $*$ * 
Research in Autism Spectrum Disorders doi:10.1016/j.rasd.2014.05.018

As follow-up analyses for the three-way interaction, a simple interaction analysis

was conducted for each intensity condition. The results revealed that the simple

353 interactions between group and presentation condition were significant under the $52 \%$ intensity condition $(F(1,60)=6.93, p=0.01)$ but not under the $80 \%(F(1,60)=0.46, p$ $>0.10)$ and $108 \%$ intensity conditions $(F(1,60)=1.52, p>0.10)$. A follow-up simple-simple main-effect analysis of group under the $52 \%$ intensity condition revealed

357 that typically developing controls perceived more exaggerated images than did

358 individuals with ASD under the dynamic condition $(F(1,120)=6.76, p=0.01)$ but not

359 under the static condition $(F(1,120)=0.08, p>0.10)$.

361 findings (Uono et al., 2010; Yoshikawa \& Sato, 2008), a follow-up analysis was

362 conducted for each group and intensity. For the control group, the simple-simple main

363 effects of presentation were significant under all intensity conditions $(52 \%: F(1,60)=$

$36434.03, p<0.01 ; 80 \%: F(1,60)=5.21, p=0.03 ; 108 \%: F(1,60)=22.29, \quad p<0.01)$.

365 For the ASD group, the simple-simple main effects of presentation were significant

366 under all intensity conditions $(52 \%: F(1,60)=4.46, p=0.04 ; 80 \%: F(1,60)=10.50, p$

$367<0.01 ; 108 \%: F(1,60)=8.88, p<0.01)$. In sum, the results indicated that both control 
Research in Autism Spectrum Disorders doi:10.1016/j.rasd.2014.05.018

368

369

370

371

372

373

374

376

377

378

379

380

381

382

383

384

and ASD groups perceived the final dynamic facial expression images to be more

exaggerated than the static expressions under all intensity conditions.

The correlation between the degree of RM and the CARS score was significant under the $52 \%(r(10)=-0.71, p<0.05)$ but not the $80 \%(r(11)=0.33, p>0.10)$ or $108 \%(r(11)=0.14, p>0.10)$ condition, indicating that the greater the reduction in RM for subtle dynamic facial expressions, the more severe the extent of social dysfunction in that ASD individual (see Fig. 3).

$* * * * * * * * * * * * * * * * * * * * * * *$

Place Fig. 3 about here

**************************

\section{Discussion}

Our results indicated that both control and ASD groups perceived the final

images in dynamic facial expressions to be more exaggerated than static facial

expressions. These results support our first prediction and replicate previous findings

showing the existence of RM for dynamic facial expressions in individuals without

(Yoshikawa \& Sato, 2008) and with ASD (Uono et al., 2010). 
Research in Autism Spectrum Disorders doi:10.1016/j.rasd.2014.05.018

expressions with subtle emotion are presented, typically developing controls perceive

more exaggerated images than do individuals with ASD. This group difference is in line

processing of subtle emotional expressions than for intense emotional expressions

390 (Ambadar et al., 2005; Bould \& Morris, 2008; Bould et al., 2008). The results of the

391 present study are also consistent with the impaired recognition of dynamic facial

paradigm, using RM, this is the first study to show the compromised processing of

suggest that individuals with ASD and typically developing individuals may see their 
Research in Autism Spectrum Disorders doi:10.1016/j.rasd.2014.05.018

403 the degree of social dysfunction in ASD individuals. As it is difficult to detect emotion

404 in subtle emotional facial expressions, the more exaggerated perceptions of subtle

405 dynamic facial expressions, shown by typically developing individuals in comparison

406 with individuals with ASD, may play a crucial role in difficulties experienced by the

407 latter group with regard to efficiently extracting emotional meaning from faces.

408 Consequently, the reduced RM for dynamic facial expressions reflecting subtle

409 emotions may prevent individuals with ASD from noticing the subtle emotional changes

410 of others and regulating their own behaviors appropriately.

412 contaminated the processing of the stimuli by individuals with ASD. However,

413 participants were asked to exactly match the image in the response window with the last

414 image of the stimulus. The presentation time for the last image (10 ms) was identical

415 across dynamic conditions. The performance of the ASD group was comparable to that

416 of the control group under the $80 \%$ and $108 \%$ dynamic conditions. Furthermore, no

417 difference in the performance of the groups was found under the $52 \%$ static condition,

418 which used the same presentation time as under the dynamic condition. Based on these

419 results, the short presentation time under the 52\% condition cannot explain the reduced

$420 \mathrm{RM}$ for subtle dynamic facial expressions in individuals with ASD. 
Research in Autism Spectrum Disorders doi:10.1016/j.rasd.2014.05.018

subtle dynamic facial expressions is important for understanding the nature of impaired

social interactions and emotional expression processing in ASD. Difficulty with facial

430 Braverman et al., 1989; Castelli, 2005; Celani et al., 1999; Grossman et al., 2000) facial

431 expressions with relatively intense emotions have reported conflicting findings.

432 Emotional communication in daily life is mainly based on dynamic facial cues. Facial expressions are often displayed with subtle intensity (Ekman, 2003; Motley \& Camden,

434 1988). Based on the results of the present study, the use of dynamic facial expressions depicting subtle emotion reveals impairments in the emotional communication of

436 people with ASD, even in experimental settings. The less exaggerated perception of

437 subtle dynamic facial expressions may explain the discrepancy between experimental 
Research in Autism Spectrum Disorders doi:10.1016/j.rasd.2014.05.018

439 used dynamic facial expressions depicting intense emotion. The use of subtle dynamic

440 facial expressions may be useful for revealing deficits in other components of the

441 processing of dynamic facial expressions among those with ASD (e.g., recognition,

442 physiological responses, and subjective feelings).

443 Impairment of low-level and biological motion processing might explain

444 reduced RM to subtle dynamic facial expressions in ASD, as individuals who are at risk

445 for the impairment of motion processing show reduced RM (Taylor \& Jacobson, 2010).

446 There is evidence that individuals with ASD have impairments in the perception of

447 biological motion depicting human actions (Blake, Turner, Smoski, Pozdol, \& Stone,

448 2003), particularly emotional actions (Hubert et al., 2007; Moore, Hobson, \& Lee 1997).

449 Moore et al. (1997) found that few children with ASD could correctly recognize

450 biological motion when stimuli were presented briefly, although their performance did

451 not significantly differ from that of children with mental retardation. Furthermore,

452 Atkinson (2009) demonstrated that impaired recognition of biological motion depicting

453 emotional actions was associated with a deficit in low-level motion processing in ASD,

454 and recently, individual differences have been reported in the degree of this impairment

455 (Milne et al., 2002; Pellicano, Gibson, Maybery, Durkin, \& Badcock, 2005). Based on

456 these findings, the reduced RM in dynamic facial expressions with subtle, but not 
Research in Autism Spectrum Disorders doi:10.1016/j.rasd.2014.05.018

457 intense, emotion might reflect variability in the impairment of low-level and biological

458 motion processing. Subtle expressions are more likely to reveal varying levels of

459 impairment in dynamic facial expression processing.

460 Our current findings provide insights into the neural mechanisms involved in

461 processing of dynamic facial expressions. Previous studies reported reductions in brain

462 activation of ASD individuals in response to dynamic facial expressions (Pelphrey et al.,

463 2007; Sato et al., 2012). The brain regions affected were the STS/MTG and IFG, which

464 are associated with processing of the dynamic aspects of social stimuli (Allison et al.,

465 2000; Iacoboni, 2005). Moreover, it has been suggested that these two regions are

466 directly connected (Catani, Howard, Pajevic, \& Jones, 2002; Rilling et al., 2008;

467 Thiebaut de Schotten et al., 2011). Sato et al. (2012) showed that effective bidirectional

468 connectivity in the primary visual cortex-STS/MTG-IFG circuit is enhanced during

469 observation of dynamic versus static facial expressions in typically developing

470 individuals but not in those with ASD. In agreement with previous neuroimaging results,

471 the current findings indicating diminished perception of emotional intensity in ASD

472 individuals suggest that bidirectional information flow may play an important role in the

473 enhancement of the perception of emotional intensity in dynamic facial expressions.

474 The work of a previous behavioral study showing that facial imitation is associated with 
Research in Autism Spectrum Disorders doi:10.1016/j.rasd.2014.05.018

475 IFG function (Iacoboni, 2005), and facilitates the recognition of dynamic facial

476 expressions (Niedenthal, Brauer, Halberstadt, \& Innes-Ker, 2001), encourages us to

477 speculate that feedback input from the IFG and the STS to the visual cortex modulates

478 the subjective perception of dynamic facial expressions. Our results did not support our third prediction, namely that individuals with

480 ASD would show stronger RM than would controls in response to extreme dynamic

481 facial expressions. There might be several reasons for this result. First, the clear RM in

482 typically developing individuals might mask any group difference. Yoshikawa and Sato

483 (2008) noted that RM for dynamic facial expressions among typically developing

484 individuals is suppressed at $100 \%$ intensity. In the present study, it is possible that facial expressions with intense emotion (108\%) might seem equally natural to individuals with

486 and without ASD. Using even more exaggerated facial expressions as stimuli may still

487 induce a group difference in RM. Second, the speed of movement (10 ms per frame) might affect RM under the $108 \%$ condition. In research conducted by Yoshikawa and Sato, slowing down the presentation of dynamic facial expressions to $40 \mathrm{~ms}$ per frame

490 induced a reduction in RM, particularly when facial expressions with intense emotion

491 were used (Experiment 1). Tardif et al. (2007) found that slowing down presentation

492 improved recognition of dynamic facial expression in individuals with ASD but not in 
Research in Autism Spectrum Disorders doi:10.1016/j.rasd.2014.05.018

493 typically developing individuals. Thus, slowing down the presentation of dynamic facial

494 expressions with intense emotions might reveal a difference in RM between individuals

495 with and without ASD.

496 It should be acknowledged that this study had several limitations. First, the

497 dynamic facial expressions used in the present study represented a linear transition

498 developed using a computer morphing technique because this approach is advantageous

499 for controlling the amount of change and reducing the noise. However, actual facial

500 expressions would differ from the present stimuli in terms of the pattern of kinematics

501 in each facial feature. It may be helpful to use real dynamic facial expressions to further

502 elucidate the deficits in RM for dynamic facial expressions. Second, the present study

503 did not address the possibility that clinical symptoms other than ASD also affected

504 perceptions of subtle dynamic facial expressions. Although the participants with ASD in

505 the present study did not meet the criteria for neurological or other psychiatric disorders,

506 previous studies have found that individuals with ASD have high rates of associated

507 psychiatric problems, including anxiety and depression (e.g., de Bruin, Ferdinand,

508 Meester, de Nijs, \& Verheij, 2007). Interestingly, recent behavioral studies also suggest

509 that the extent of co-morbid alexithymia contributes to emotional recognition

510 impairments in ASD individuals (see Bird and Cook (2013) for a review). Promising 
Research in Autism Spectrum Disorders doi:10.1016/j.rasd.2014.05.018

511 directions for further research include analysis of the effects of psychological states and

512 traits on individuals with and without ASD.

513

514 5. Conclusions

515 In summary, the present study showed that individuals with ASD perceived the

516 final images in dynamic facial expressions to be more exaggerated than static facial

517 expressions. However, when they observed facial expressions with subtle emotion,

518 typically developing controls perceived them as more exaggerated than did individuals

519 with ASD under dynamic but not under static conditions. Emotional communication in

520 daily life is based principally on dynamic facial cues, and facial expressions are often

521 subtle. It is possible that individuals with ASD, with their reduced perception of

522 emotional intensity, have a reduced ability to detect subtle changes in other people's

523 facial expressions for use as information for adaptive behavioral responses. 
Research in Autism Spectrum Disorders doi:10.1016/j.rasd.2014.05.018

\section{Acknowledgements}

526 We would like to thank Dr. Sayaka Yoshimura for recruiting participants. We

527 are grateful to both our volunteer study participants and their parents.

528 
Research in Autism Spectrum Disorders doi:10.1016/j.rasd.2014.05.018

531 11J05000); the JSPS Funding Program for Next Generation World-Leading Researchers

532 (LZ008); grant support for graduate students awarded by the Kyoto University GCOE

533 program (Revitalizing Education for Dynamic Hearts and Minds); the Organization for

534 Promoting Developmental Disorder Research; and the Benesse Corporation. No funding

535 source had any involvement in study design; in the collection, analysis, or interpretation

536 of data; in the writing of the report; or in the decision to submit the article for

537 publication. 
Research in Autism Spectrum Disorders doi:10.1016/j.rasd.2014.05.018

540 Adolphs, R., Sears, L., \& Piven, J. (2001). Abnormal processing of social information

541 from faces in autism. Journal of Cognitive Neuroscience, 13, 232-240.

542 Allison, T., Puce, A., \& McCarthy, G. (2000). Social perception from visual cues: Role of the STS region. Trends in Cognitive Sciences, 4, 267-278.

544 Ambadar, Z., Schooler, J.W., \& Cohn, J.F. (2005). Deciphering the enigmatic face: The

545 importance of facial dynamics in interpreting subtle facial expressions.

$546 \quad$ Psychological Science, 16, 403-410.

547 American Psychiatric Association (2000). Diagnostic and statistical manual for mental

548 disorders (DSM-IV-TR). Washington, DC: Author.

549 Ashwin, C., Chapman, E., Colle, L., \& Baron-Cohen, S. (2006). Impaired recognition of 550 negative basic emotions in autism: A test of the amygdala theory. Social Neuroscience, 1, 349-363.

552 Atkinson, A. P. (2009). Impaired recognition of emotions from body movements is associated with elevated motion coherence thresholds in autism spectrum disorders. Neuropsychologia, 47, 3023-3029.

Azuma, H., Ueno, K., Fujita, K., Maekawa, H., Ishikuma, T., \& Sano, H. (1998). 
Research in Autism Spectrum Disorders doi:10.1016/j.rasd.2014.05.018

Nihon-Bunka-Kagaku-sha.

558

Bird, G., \& Cook, R. (2013). Mixed emotions: The contribution of alexithymia to the emotional symptoms of autism. Translational Psychiatry, 3, e285.

Blake, R., Turner, L.M., Smoski, M.J., Pozdol, S.L., \& Stone, W.L. (2003). Visual recognition of biological motion is impaired in children with autism. Psychological Science, 14, 151-157.

Bould, E., \& Morris, N. (2008). Role of motion signals in recognizing subtle facial expressions of emotion. British Journal of Psychology, 99, 167-189.

Bould, E., Morris, N., \& Wink, B. (2008). Recognising subtle emotional expressions: children with pervasive developmental disorders. Journal of Autism and Developmental Disorders, 19, 301-316. rates of psychiatric co-morbidity in PDD-NOS. Journal of Autism and Developmental Disorders, 37, 877-886.

573 Calder, A. J., Lawrence, A. D., \& Young, A. W. (2001). Neuropsychology of fear and loathing. Nature Reviews Neuroscience, 2, 352-363. 
Research in Autism Spectrum Disorders doi:10.1016/j.rasd.2014.05.018

575 Castelli, F. (2005). Understanding emotions from standardized facial expressions in autism and normal development. Autism, 9, 428-449.

577 Catani M., Howard, R. J., Pajevic, S., \& Jones, D. K. (2002). Virtual in vivo interactive dissection of white matter fasciculi in the human brain. Neuroimage, 17, 77-94.

579 Celani, G., Battacchi, M. W., \& Arcidiacono, L. (1999). The understanding of the emotional meaning of facial expressions in people with autism. Journal of Autism and Developmental Disorders, 29, 57-66.

582 Corona, R., Dissanayake, C., Arbelle, S., Wellington, P., \& Sigman, M. (1998). Is affect aversive to young children with autism? Behavioral and cardiac responses to experimenter distress. Child Development, 69, 1494-1502.

Ekman, P. (2003). Emotions revealed. New York: Times Books

Ekman, P. and Friesen, W.V. (1975). Unmasking the face: A guide to recognizing emotions from facial clues. Englewood Cliffs, NJ: Prentice-Hall.

Ekman, P., \& Friesen, W.V. (1976). Pictures of facial affect. Palo Alto, CA: Consulting Psychologists Press.

590 Freyd, J. J., \& Finke, R. A. (1984). Representational momentum. Journal of Experimental Psychology: Learning, Memory and Cognition, 10, 126-132.

592 Frith, U., \& Frith, C. D. (2003). Development and neurophysiology of mentalizing. 
Research in Autism Spectrum Disorders doi:10.1016/j.rasd.2014.05.018

Proceedings of the Royal Society of London B: Biological Sciences, 358, 459-473.

594 Fujita, K., Maekawa, H., Dairoku, H., \& Yamanaka, K.. (2006). Japanese Wechsler adult intelligence scale-III. Tokyo: Nihon-Bunka-Kagaku-sha.

596 Gepner, B., Deruelle, C., \& Grynfeltt, S. (2001). Motion and emotion: A novel approach to the study of face processing by young autistic children. Journal of Autism and Developmental Disorders, 31, 37-45.

599 Grossman, J. B., Klin, A., Carter, A.S., \& Volkmar, F. R. (2000). Verbal bias in

600 recognition of facial emotions in children with Asperger syndrome. Journal of Child

601 Psychology and Psychiatry, 41, 369-379.

602

Haxby, J. V., Hoffman, E. A., \& Gobbini M. I. (2000). The distributed human neural system for face perception. Trends in Cognitive Sciences, 4, 223-233.

604 Hubbard, T. L. (1990). Cognitive representation of linear motion: Possible direction and 605 gravity effects in judged displacement. Memory and Cognition, 18, 299-309.

606 Hubert, B., Wicker, B., Moore, D. G., Monfardini, E., Duverger, H., Da Fonseca, D., et al. (2007). Recognition of emotional and non-emotional biological motion in individual with Autistic Spectrum Disorders. Journal of Autism and Developmental Disorders, 37, 1386-1392.

610 Hudson, M., Liu, C. H., \& Jellema, T. (2009). Anticipating intentional actions: The 
Research in Autism Spectrum Disorders doi:10.1016/j.rasd.2014.05.018

611 effect of eye gaze direction on the judgment of head rotation. Cognition, 112,

$612 \quad 423-434$

613 Iacoboni, M. (2005). Neural mechanisms of imitation. Current Opinion in Neurobiology,

$614 \quad 15,632-637$.

615 Kessels, R. P. C., Spee, P., \& Hendriks, A. W. (2010). Perception of dynamic facial

616 emotional expressions in adolescents with autism spectrum disorders (ASD).

617 Translational Neuroscience, 1, 228-232.

618 Kirk, R. E. (1995). Experimental design: Procedures for the behavioral sciences (3rd

619 ed.). Pacific Grove, CA: Brooks/Cole.

620 Kodama, H., Shinagawa, F., \& Motegi, M. (1982). Japanese Wechsler intelligence scale

621 for children-revised. Tokyo: Nihon-Bunka-Kagaku-sha.

622 Koyama, T., Tachimori, H., Osada, H., Takeda, T., \& Kurita, H. (2007). Cognitive and

623 symptom profiles in Asperger's syndrome and high-functioning autism. Psychiatry

624 and Clinical Neuroscience, 61, 99-104.

625 LaBar, K. S., Crupain, M. J., Voyvodic, J. T., \& McCarthy, G. (2003). Dynamic

626 perception of facial affect and identity in the human brain. Cerebral Cortex, 13,

$627 \quad 1023-1033$.

628 Mesibov, G. B., Schopler, E., Schaffer, B., \& Michal, N. (1989). Use of the Childhood 
Research in Autism Spectrum Disorders doi:10.1016/j.rasd.2014.05.018

Autism Rating Scale with autistic adolescents and adults. Journal of the American

630 Academy of Child and Adolescent Psychiatry, 28, 538-541.

631 Milne, E., Swettenham, J., Hansen, P., Campbell, R., Jeffries, H., \& Plaisted, K. (2002).

632 High motion coherence thresholds in children with autism. Journal of Child Psychology and Psychiatry, 43, 255-263.

634 Moore, D. G., Hobson, R. P., \& Lee, A. (1997). Components of person perception: An investigation with autistic, non-autistic retarded and typically developing children and adolescents. British Journal of Developmental Psychology, 15, 401-423.

Motley, M., \& Camden, C. (1988). Facial expression of emotion: A comparison of posed expressions versus spontaneous expressions in an interpersonal communications setting. Western Journal of Speech Communication, 52, 1-22.

640 Mukaida, S., Kamachi, M., Kato, T., Oda, M., Yoshikawa, S., \& Akamatsu, S. (2000). Foolproof utilities for facial image manipulation. Kyoto: ATR [unpublished computer software].

643 Niedenthal, P. M., Brauer, M., Halberstadt, J. B., \& Innes-Ker, Å. H. (2001). When did

644 her smile drop? Facial mimicry and the influences of emotional state on the 645 detection of change in emotional expression. Cognition and Emotion, 15, 853-864.

646 Oldfield, R. C. (1971). The assessment and analysis of handedness: The Edinburgh 
Research in Autism Spectrum Disorders doi:10.1016/j.rasd.2014.05.018

inventory. Neuropsychologia, 9, 97-113.

648 Pellicano, E., Gibson, L., Maybery, M., Durkin, K., \& Badcock, D. R. (2005). Abnormal

649 global processing along the dorsal visual pathway in autism: A possible mechanism

650 for weak visuospatial coherence? Neuropsychologia, 43, 1044-1053.

651 Pelphrey, K. A., Morris, J. P., McCarthy, G., \& Labar, K. S. (2007). Perception of dynamic changes in facial affect and identity in autism. Social Cognitive and Affective. Neuroscience, 2, 140-149.

654

Rilling, J.K., Glasser, M.F., Preuss, T. M., Ma, X., Zhao, T., Hu, X., et al. (2008). The evolution of the arcuate fasciculus revealed with comparative DTI. Nature Neuroscience, 11, 426-428.

Rutherford, M. D., \& McIntosh, D. N. (2007). Rules versus prototype matching: Strategies of perception of emotional facial expressions in the autism spectrum. Journal of Autism and Developmental Disorders, 37, 187-196.

660 Sato, W., Kochiyama, T., Yoshikawa, S., Naito, E., \& Matsumura, M. (2004). Enhanced neural activity in response to dynamic facial expressions of emotion: An fMRI study. Brain Research: Cognitive Brain Research, 20, 81-91.

663 Sato, W., Toichi, M., Uono, S., \& Kochiyama, T. (2012). Impaired social brain network 
Research in Autism Spectrum Disorders doi:10.1016/j.rasd.2014.05.018

Neuroscience, 13, 99.

666 Sato, W., \& Yoshikawa, S. (2007a). Enhanced experience of emotional arousal in

667 response to dynamic facial expressions. Journal of Nonverbal Behavior, 31,

668 $119-135$.

669

Sato, W., \& Yoshikawa, S. (2007b). Spontaneous facial mimicry in response to dynamic

670 facial expressions. Cognition, 104, 1-18.

671

Schopler, R., Reichler, R. J., \& Renner, B. R. (1986). The Childhood Autism Rating

672 Scale (CARS) for diagnostic screening and classification of autism. New York:

673 Irvington.

674

Shinagawa, F., Kobayashi, D., Fujita, K., \& Maekawa, H. (1990). Japanese Wechsler adult intelligence scale-revised. Tokyo: Nihon-Bunka-Kagaku-sha.

676

Sigman, M. D., Kasari, C., Kwon, J. H., \& Yirmiya, N. (1992). Responses to the negative emotions of others by autistic, mentally retarded, and normal children.

678 Child Development, 63, 796-807.

679

Tardif, C., Lainé, F., Rodriguez, M., \& Gepner, B. (2007). Slowing down presentation

680 of facial movements and vocal sounds enhances facial expression recognition and induces facial-vocal imitation in children with autism. Journal of Autism and 
Research in Autism Spectrum Disorders doi:10.1016/j.rasd.2014.05.018

683 Taylor, N., \& Jacobson, L. (2010). Representational momentum in children born

684 preterm and at term. Brain and Cognition, 72, 464-471.

685 Thiebaut de Schotten, M., Ffytche, D. H., Bizzi, A., Dell'Acqua, F., Allin, M., Walshe,

686 M., et al. (2011). Atlasing location, asymmetry and inter-subject variability of white

687 matter tracts in the human brain with MR diffusion tractography. NeuroImage, 54,

$688 \quad 49-59$.

689 Uono, S., Sato, W., \& Toichi, M. (2010). Brief report: Representational momentum for

690 dynamic facial expressions in pervasive developmental disorder. Journal of Autism

691 and Developmental Disorders, 40, 371-377.

692 Uono, S., Sato, W., \& Toichi, M. (2011). The specific impairment of fearful expression recognition and its atypical development in pervasive developmental disorder.

694 Social Neuroscience, 6, 452-463.

695

Uono, S., Sato, W., \& Toichi, M. (2013). Common and unique impairments in

696 facial-expression recognition in pervasive developmental disorder-not otherwise

697 specified and Asperger's disorder. Research in Autism Spectrum Disorders, 7,

698 $361-368$

699 Warren, G., Schertler, E., \& Bull, P. (2009). Detecting deception from emotional and unemotional cues. Journal of Nonverbal Behavior, 33, 59-69. 
Research in Autism Spectrum Disorders doi:10.1016/j.rasd.2014.05.018

701 Yirmiya, N., Kasari, C., Sigman, M., \& Mundy, P. (1989). Facial expressions of affect in

702 autistic, mentally retarded and normal children. Journal of Child Psychology and

$703 \quad$ Psychiatry, 30, 725-735.

704 Yoon, K. L., Joormann, J., \& Gotlib, I. H. (2009). Judging the intensity of facial

705 expressions of emotion: Depression-related biases in the processing of positive

706 affect. Journal of Abnormal Psychology, 118, 223-228.

707 Yoshikawa, S., \& Sato, W. (2008). Dynamic facial expressions of emotion induce

708 representational momentum. Cognitive, Affective \& Behavioral Neuroscience, 8,

$709 \quad 25-31$.

710 
Research in Autism Spectrum Disorders doi:10.1016/j.rasd.2014.05.018

711

\section{Table 1}

Mean (with SE) intensities of the selected images in individuals with ASD and typically developing controls $(\mathrm{CON})$

\begin{tabular}{|l|l|l|l|l|}
\hline Group & Presentation & Intensity & \\
\cline { 3 - 5 } & & $52 \%$ & $80 \%$ & $108 \%$ \\
\hline ASD & Dynamic & $59.3(1.5)$ & $88.1(1.3)$ & $115.4(1.6)$ \\
& Static & $57.0(1.2)$ & $82.7(1.3)$ & $108.7(1.6)$ \\
\hline \multirow{2}{*}{ CON } & Dynamic & $63.0(0.9)$ & $89.5(1.2)$ & $119.4(2.1)$ \\
& Static & $56.7(1.3)$ & $85.7(1.5)$ & $108.8(1.5)$ \\
\hline
\end{tabular}

712

713 
Research in Autism Spectrum Disorders doi:10.1016/j.rasd.2014.05.018

714 Figure Captions

715 Fig. 1 a) Examples of the morphing image sequence for dynamic facial expressions of

716 emotion. b) Final image of dynamic facial expressions under each intensity condition.

718 Fig. 2 Mean ratio between the intensity of the selected and presented images under each

719 condition. The asterisk represents a significant interaction between group and

720 presentation, indicating reduced RM for subtle dynamic facial expressions in ASD.

721 Error bars show the SE.

722

723 Fig. 3 Correlation between degree of RM and CARS scores under the $52 \%$ condition.

724 Black and white diamonds show participants with Asperger's disorder and PDD-NOS,

725 respectively.

726

727

728

729

730 
Research in Autism Spectrum Disorders doi:10.1016/j.rasd.2014.05.018

732 Fig. 1

a)

\section{Dynamic}

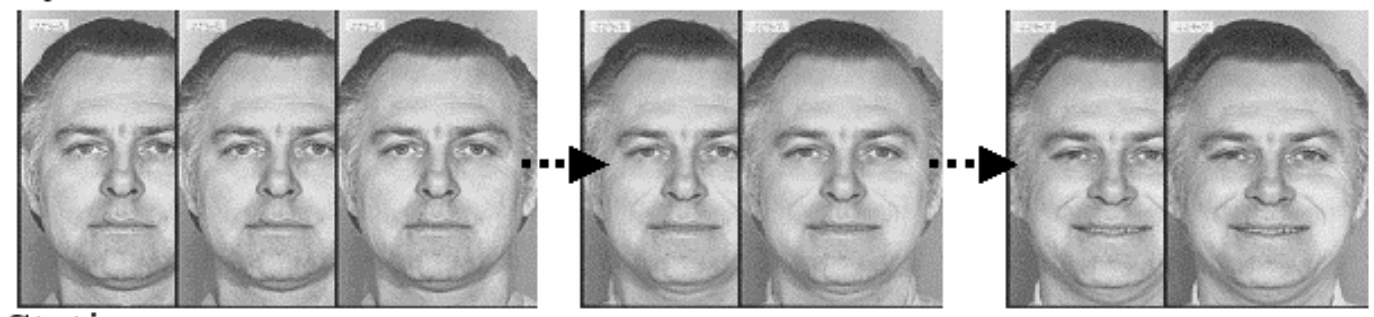

\section{Static}
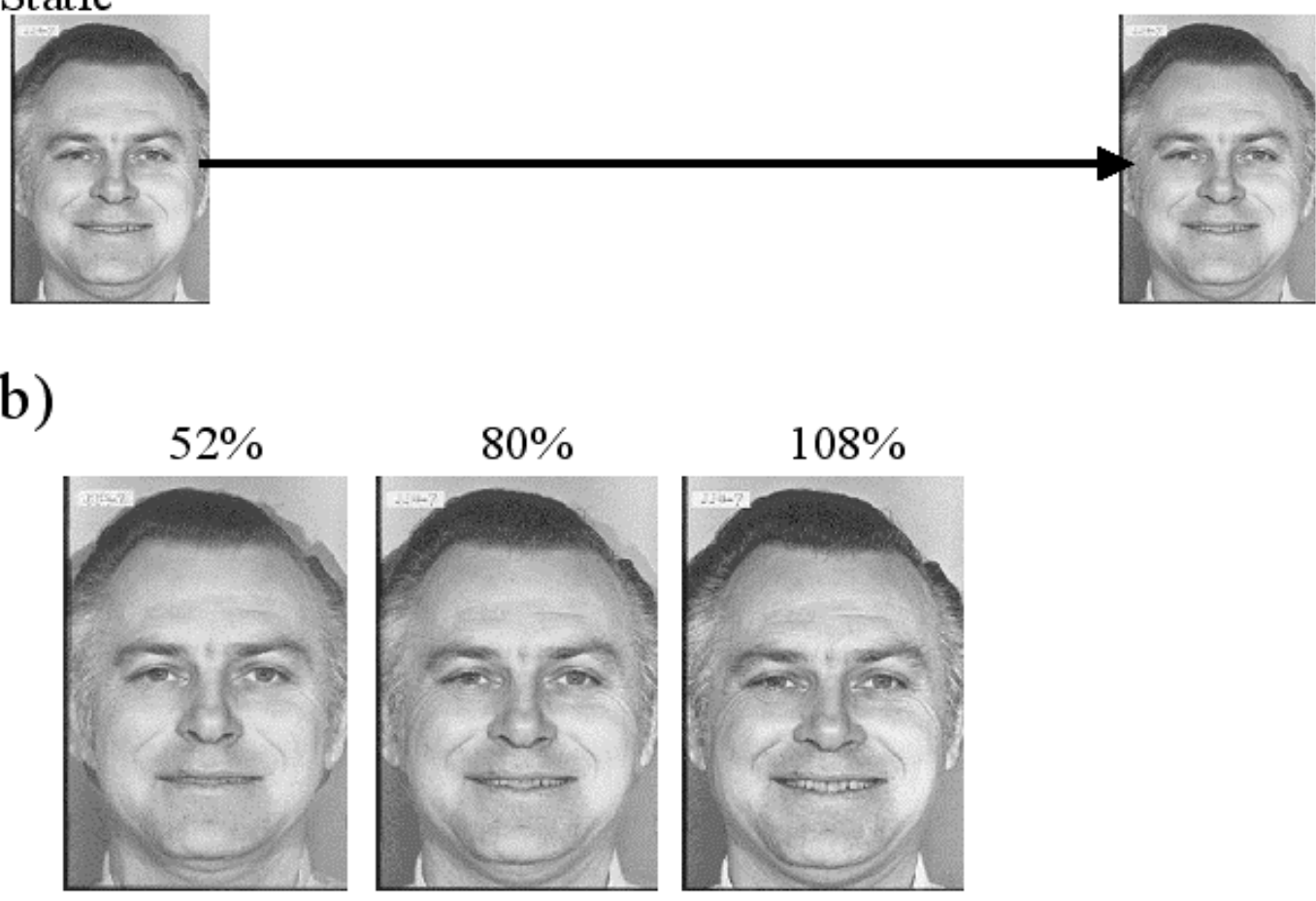

b)

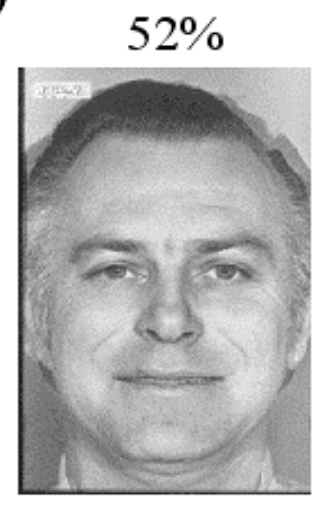


Research in Autism Spectrum Disorders doi:10.1016/j.rasd.2014.05.018

Fig. 2

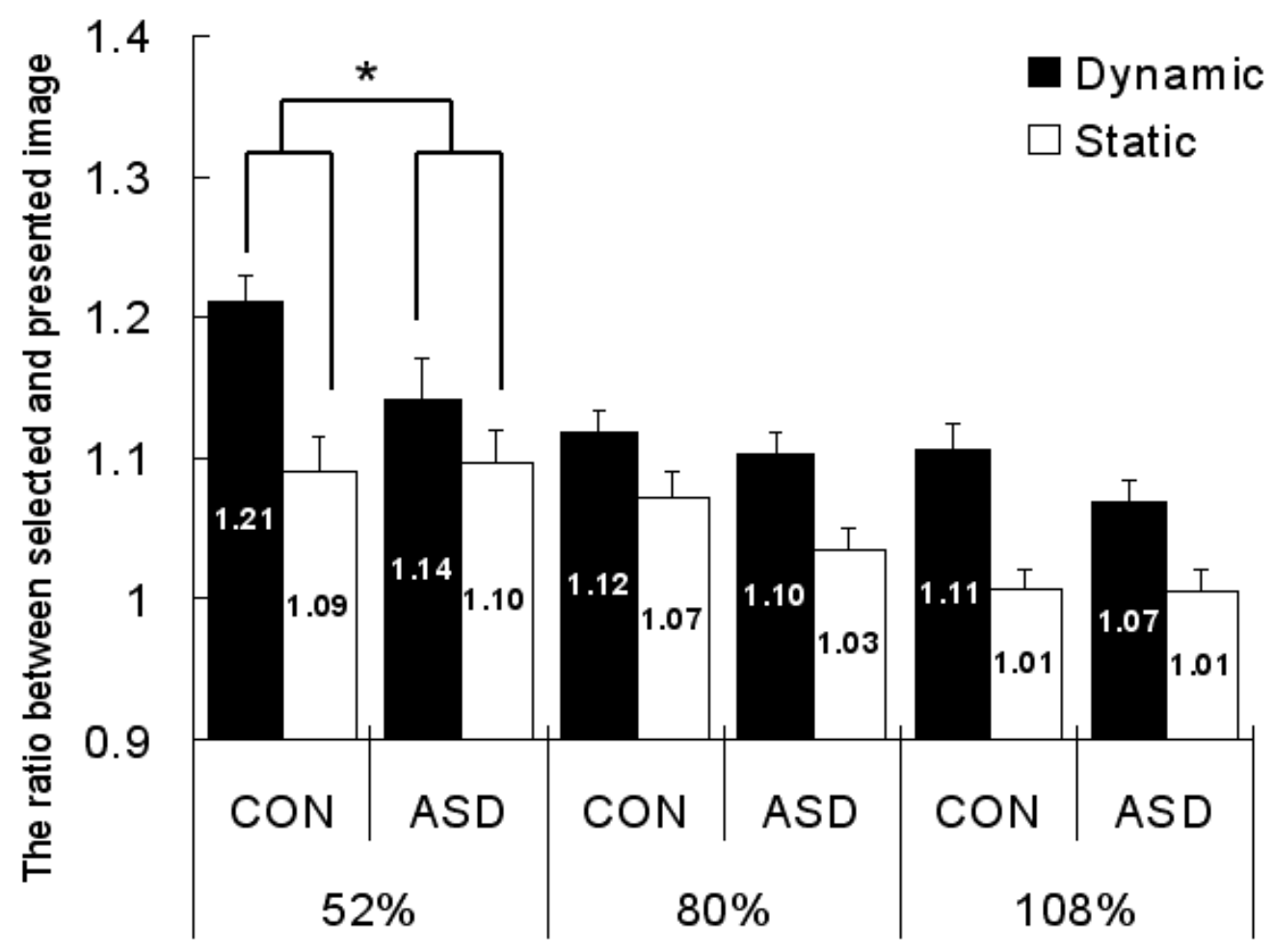

736 
Research in Autism Spectrum Disorders doi:10.1016/j.rasd.2014.05.018

738 Fig. 3

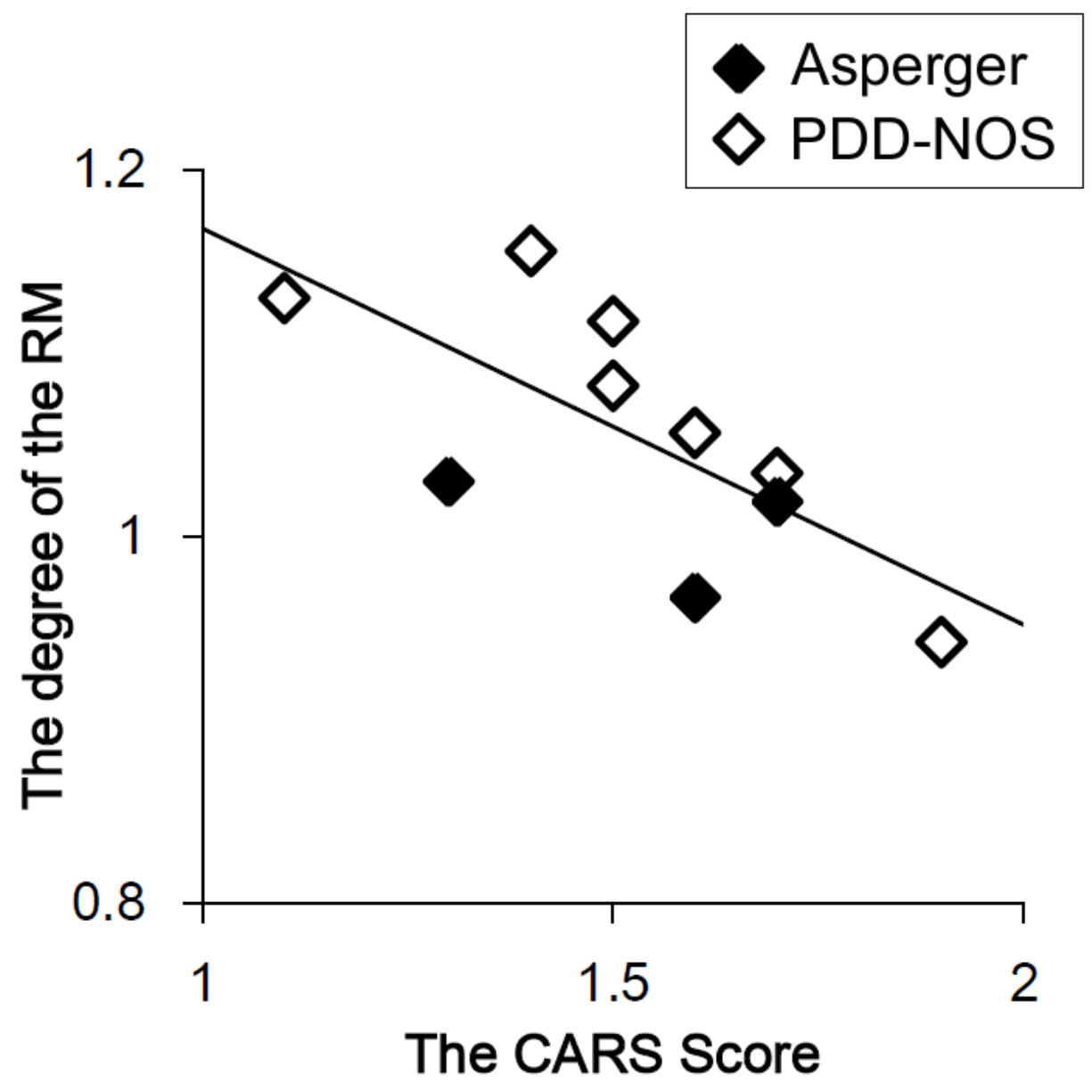

